\title{
Multicentre study of Wilm's tumours treated by different therapeutic strategies in two different countries
}

\author{
Mohamed A. Elmagd Salem ${ }^{a, b}$, Yoshida Kinoshita ${ }^{a}$, Mohamed Abdelkhader ${ }^{\mathrm{e}}$, \\ Hamza A. Hamza ${ }^{c}$ and Amany M. Ali
}

\begin{abstract}
Background and purpose According to the treatment of Wilm's tumours, two different therapeutic strategies were established in the second half of the last century. Both National Wilm's Tumour Study (NWTS) group and the International Society of Paediatric Oncology (SIOP) have helped to improve the clinical management and outcome of patients with Wilm's tumours. In this study, we compared three groups of patients with Wilm's tumours from different racial backgrounds and therapeutic strategies.
\end{abstract}

Patients and methods A clinicopathological review was carried out for $\mathbf{4 0}$ patients treated at Kyushu University Hospital in Japan from 1960 to 2006 according to NWTS, 79 patients treated at the South Egypt Cancer Institute from 2002 to 2009 according to the SIOP protocol and 33 patients treated at the Pediatric Surgery Department of Assiut University Hospital from 2000 to 2009 according to the NWTS protocol.

Results In the Kyushu University group, a favourable histology was diagnosed in $\mathbf{8 0} \%$ of patients and an unfavourable histology in $\mathbf{2 0 \%}$ of patients. The distribution of the clinical stage was as follows: $I=40 \%, I I=22.5 \%$, $\mathrm{III}=\mathbf{2 2 . 5} \%$, IV=7.5\% and $\mathrm{V}=\mathbf{7 . 5} \%$. The 5 -year overall survival was $\mathbf{8 2 . 5} \%$, with the 5 -year stage-related survival as follows: $\mathrm{I}=93.7 \%, \mathrm{II}=83.3 \%, \mathrm{III}=62.5 \%, \mathrm{IV}=33.3 \%$ and $\mathrm{V}=100 \%$. In the South Egypt Cancer Institute group, favourable histology was diagnosed in $89.9 \%$ of patients, unfavourable histology in $\mathbf{8 . 8 \%}$ of patients and benign disease in $1.3 \%$ of patients. The distribution of the clinical stage (before and after chemotherapy) was as follows: $\mathrm{I}=\mathbf{2 5 . 3} \% \rightarrow \mathbf{5 0 \%}, \mathrm{II}=\mathbf{1 9} \% \rightarrow \mathbf{2 0 \%}, \mathrm{III}=\mathbf{3 5 . 4} \rightarrow \mathbf{1 5} \%$, IV $=12.5 \% \rightarrow 5 \%$ and $V=7.6 \% \rightarrow 7.6 \%$. No case of

\section{Introduction}

Renal tumours account for about $12 \%$ of childhood tumours [1]. Wilm's tumour is the most common renal tumour of infancy and childhood. Its incidence varies according to race, from 2.5 per million Chinese to 10.9 per million American black children [2]. It is most usually a unilateral disease, but in $5-10 \%$ of cases, both kidneys are affected [3].

Over the past two decades, there has been a significant improvement in the survival of children with cancer in general and those with Wilm's tumours in particular; this is largely attributed to multicentric prospective studies carried out by the National Wilm's Tumour Study (NW'S ) group and the International Society of Paediatric Oncology (SIOP) [4].

The timing of surgery in relation to chemotherapy is still controversial in the management of patients with Wilm's tumours. According to the NWTS protocol, these intraoperative tumour rupture was recorded. The overall 5-year survival rate of $84 \%$ with a 5 -year stage-related survival was as follows: $I=92 \%, I I=80 \%, I I I=50 \%$, $\mathrm{IV}=\mathbf{3 1} \%$ and $\mathrm{V}=\mathbf{3 0} \%$. In the Assiut University group, a favourable histology was diagnosed in $\mathbf{7 9} \%$ of patients and an unfavourable histology in $9 \%$ of patients. The distribution of the clinical stage was as follows: $I=39.4 \%$, $\mathrm{II}=\mathbf{2 1 . 2} \%, \mathrm{III}=\mathbf{2 1 . 2} \%, \mathrm{IV}=12 \%$ and $\mathrm{V}=6 \%$. The overall 5-year survival rate was $\mathbf{7 7} \%$ and the 5 -year stage-related survival was as follows: $I=85 \%, I I=75 \%, I I I=52 \%$, $\mathrm{IV}=33 \%$ and $\mathrm{V}=33 \%$.

Conclusion The Egyptian patients with Wilm's tumour presented at a younger age and had less incidence of an unfavourable histology compared with Japanese patients. Preoperative chemotherapy increased the rate of stage I disease and decreased the incidence of intraoperative tumour rupture and relapse. However, there was a possibility that chemotherapy had been administered in the case of benign disease. Ann Pediatr Surg 9:25-30 (c) 2013 Annals of Pediatric Surgery.

Annals of Pediatric Surgery 2013, 9:25-30

Keywords: intraoperative tumour rupture, NWTS, SIOP, Wilm's tumour

a Department of Pediatric Surgery, Graduate School of Medical Sciences, Kyushu University, Fukuoka, Japan, Departments of ' Surgical Oncology, ${ }^{\mathrm{c}}$ Radiation Oncology, ${ }^{\mathrm{d} P e d i a t r i c}$ Oncology, South Egypt Cancer Institute and ${ }^{\mathrm{e}}$ Department of Pediatric Surgery, Faculty of Medicine, Assiut University, Asyut, Egypt

Correspondence to Mohamed A. Elmagd Salem, Department of Surgical Oncology, South Egypt Cancer Institute, Assiut University, Asyut, Egypt Tel: +20 100000 4857; fax: + 2088 2348609; e-mail: salem641972@homail.com

Received 19 November 2011 accepted 14 October 2012

children should be treated first by surgery, followed by chemotherapy with or without radiotherapy, depending on the stage [5]. The SIOP group, in contrast, advocates preoperative chemotherapy, followed by surgery. This approach is an attempt to decrease the size of the tumour and thus reduce the risk of intraoperative rupture; however, the survival in both groups is similar [6].

Here, we report the characteristics of Wilm's tumours and the results of the multimodality treatment in three groups of patients with Wilm's tumours from different racial backgrounds and different therapeutic strategies.

For the Kyushu University (KU) group, the NWTS approach was used during the period 1960-2006, for the Assiut University (AU) group, the NWTS approach was used during the period 2000-2009 and for the South Egypt Cancer Institute (SECI) group, the SIOP approach was used during the period 2002-2009. 


\section{Patients and methods}

For the Kyushu University Hospital group, a retrospective study was carried out in 40 patients treated according to NWTS and JWiTS (Japanese Wilm's tumour Study since 1996) group protocols during the period from 1960 to 2006. Immediate nephrectomy was performed wherever the surgeons believed that the approach was feasible; in those cases where the patient had a tumour that was deemed unresectable, and in those with bilateral, inferior vena cava malignant thrombus and distant metastasis, preoperative chemotherapy was provided.

For the AU group, a retrospective study was carried out of 33 patients treated at the Pediatric Surgery Department of Assiut University Hospital Egypt from 2000 to 2009 according to the NWTS protocol.

For the SECI, a prospective study was carried out in patients with Wilm's tumours admitted at the Paediatric Oncology Department during the period between January 2002 and January 2009 and treated according to the SIOPbased protocol; during this period, 79 patients were admitted with a clinical and radiological diagnosis of Wilm's tumour and treated according to the SIOP-based protocol.

The medical records of patients were reviewed for age at diagnosis, sex, incidence, presenting symptoms, preoperative chemotherapy treatment, type of surgery, histopathological diagnosis, stage (on the basis of the NWTS and JWiTS staging system for the Kyushu and Assiut Universities groups and the SIOP staging system for the SECI group) and final outcome.

Approval was obtained from the institute review board of the participating centers.

\section{Operative technique}

A transverse supraumbilical muscle cutting transperitoneal incision, and exploration of the liver, spleen, peritoneal surface and contralateral kidney for the presence of metastasis and a synchronous Wilm's tumour were performed. Mobilization of the colon and mesentery medially and early ligation of the vascular pedicle before extensive mobilization of the tumour is recommended if possible. Ligation of artery and vein separately and the artery before the vein, radical nephrectomy and paraaortic lymph node sampling were performed.

\section{Radiotherapy}

Radiotherapy doses and indications are as follows: $10.8 \mathrm{~Gy}$ to the flank for stage III favourable histology, I-III focal anaplasia, I-II diffuse anaplasia, I-III clear cell and 19.8 Gy to the flank for stage III diffuse anaplasia, I-III rhabdoid. Whole-abdomen radiation should be performed if there is diffuse tumour spillage, preoperative or intraperitoneal tumour rupture, peritoneal tumour seeding and cytologypositive ascites. Gross residual disease after surgery should receive a $10 \mathrm{~Gy}$ boost. In cases with lung metastasis, we administered $12 \mathrm{~Gy}$ to the whole lung in eight fractions; for brain metastasis, $30.6 \mathrm{~Gy}$ to the whole brain in 17 fractions; for liver metastasis, $19.8 \mathrm{~Gy}$ to the whole liver in 11 fractions; for bone metastasis, 25.2 Gy to the lesion plus a $3 \mathrm{~cm}$ margin and $19.8 \mathrm{~Gy}$ for unresected lymph node metastasis.

\section{Results}

A retrospective study was carried out of 179 patients with Wilm's tumour registered at the KU, AU and SECI during the study period; 73 patients were treated with primary nephrectomy according to the NWTS-based protocol in Kyushu and Assiut Universities and 79 patients were treated with preoperative chemotherapy according to the SIOP-based protocol in SECI.

\section{Kyushu University group data}

This group included 42 patients treated at the Pediatric Surgery Department, KU Hospital, Japan, during the period from 1960 to 2006. Two patients were excluded from the study because the postoperative histopathological diagnosis was renal cell carcinoma. This group was treated according to NWTS, where primary nephrectomy was performed first, followed by chemotherapy and/or radiotherapy. Some patients in this group were subjected to preoperative chemotherapy because of the presence of massive, bilateral tumours, inferior vena cava malignant thrombus or distance metastases. There were 21 males and 19 females, with a male to female ratio of $1.1: 1$. The age of the patients ranged from 2 months to 10.5 years, mean age 36 months. Nineteen patients (47.5\%) were younger than 2 years of age and $72.5 \%$ were younger than 4 years of age at the time of diagnosis. The tumour was localized in the left kidney in $22(55 \%)$ patients, the right kidney in $15(37.5 \%)$ and was bilateral in three (7.5\%). The mode of presentation in this group is shown in Table 1.

The stage distribution of patients treated in Kyushu University Hospital was as follows: 16 (40\%) patients presented with stage I disease, nine $(22.5 \%)$ presented with stage II disease, nine $(22.5 \%)$ presented with stage III disease, three (7.5\%) presented with metastatic disease and three (7.5\%) had synchronous Wilm's tumour.

The pathology of childhood renal tumours in the patients treated in $\mathrm{KU}$ is shown in Table 1; in 33 (82.5\%) patients, the histopathological diagnosis was nephroblastoma with a favourable histology and seven (17.5\%) had an unfavourable histology. Intraoperative tumour rupture occurred in six patients $(15 \%)$.

Incomplete resection was encountered in nine (23\%) of 40 patients. Seven $(77.7 \%)$ of these patients developed postoperative relapse; five $(71.5 \%)$ of these patients died because of their disease.

Among 40 patients, seven (17.5\%) died during the course of follow-up; four (57\%) of them had an unfavourable histopathology (three with a rhabdoid tumour of the kidney and one with clear cell sarcoma of the kidney).

The 5-year overall survival rate of patients treated in Kyushu University Hospital was $82.5 \%$. The stage-related survival was as follows: $93.7 \%$ for stage I, $88.8 \%$ for stage II, $66.6 \%$ for stage III, $33.3 \%$ for stage IV, and $100 \%$ for stage $\mathrm{V}$.

\section{South Egypt Cancer Institute group data}

During the period from January 2002 to January 2009, 79 patients with Wilm's tumour were admitted to the 
Table 1 Clinical characteristics and outcome of patients with Wilm's tumours under study

\begin{tabular}{|c|c|c|c|}
\hline Variables & $\begin{array}{c}\text { KU group } \\
\text { (40 patients) } \\
\text { NWTS protocol }\end{array}$ & $\begin{array}{c}\text { AU group } \\
\text { (33 patients) } \\
\text { NWTS protocol }\end{array}$ & $\begin{array}{l}\text { SECl group } \\
\text { (79 patients) } \\
\text { SIOP protocol }\end{array}$ \\
\hline \multicolumn{4}{|l|}{ Age (months) } \\
\hline $0-23$ & $19(47.5 \%)$ & $17(51.5 \%)$ & $47(59.5 \%)$ \\
\hline $24-47$ & $10(25 \%)$ & $11(33.3 \%)$ & $28(35.5 \%)$ \\
\hline$\geq 48$ & $11(27.5 \%)$ & $5(15.2 \%)$ & $4(5 \%)$ \\
\hline $\begin{array}{c}\text { Mean age } \\
\text { (years) }\end{array}$ & 3 & 2.5 & 2 \\
\hline \multicolumn{4}{|l|}{ Sex } \\
\hline Male & $21(52.5 \%)$ & $15(45.5 \%)$ & $40(50.5 \%)$ \\
\hline Female & 19 (47.5\%) & 18 (54.5\%) & 39 (49.5\%) \\
\hline \multicolumn{4}{|l|}{ Site } \\
\hline Right & 15 (37.5\%) & $13(39.4 \%)$ & $30(38 \%)$ \\
\hline Left & $22(55 \%)$ & $17(51.5 \%)$ & $43(54.4 \%)$ \\
\hline Bilateral & $3(7.5 \%)$ & $2(6 \%)$ & $6(7.6 \%)$ \\
\hline \multicolumn{4}{|l|}{ Histopathology } \\
\hline Favourable & $33(82.5 \%)$ & $30(91 \%)$ & 71 (89.9\%) \\
\hline Anaplastic & $1(2.5 \%)$ & $1(3 \%)$ & $4(5.1 \%)$ \\
\hline Rabdoid tumour & $3(7.5 \%)$ & $1(3 \%)$ & $2(2.5 \%)$ \\
\hline $\begin{array}{c}\text { Clear cell sarcoma } \\
\text { of the kideny }\end{array}$ & $3(7.5 \%)$ & $1(3 \%)$ & $1(1.3 \%)$ \\
\hline Benign disease & 0 & 0 & $1(1.3 \%)$ \\
\hline \multicolumn{4}{|l|}{ Pretreatment staging } \\
\hline Stage I & $16(40 \%)$ & 13(39.4\%) & $20(25.3 \%)$ \\
\hline Stage II & $9(22.5 \%)$ & $7(21.2 \%)$ & 15 (19\%) \\
\hline Stage III & $9(22.5 \%)$ & $7(21.2 \%)$ & $28(35.4 \%)$ \\
\hline Stage IV & $3(7.5 \%)$ & $4(12.1 \%)$ & $10(12.6 \%)$ \\
\hline Stage V & $3(7.5 \%)$ & $2(6 \%)$ & $6(7.6 \%)$ \\
\hline Tumour rupture & $6(15 \%)$ & $7(21.2 \%)$ & $0(0 \%)$ \\
\hline Incomplete resection & $9(23 \%)$ & $10(30 \%)$ & $7(8.8 \%)$ \\
\hline Recurrence & $7(18 \%)$ & $7(21.2 \%)$ & $8(10 \%)$ \\
\hline Overall survival & 33(82.5\%) & $77 \%$ & $84 \%$ \\
\hline
\end{tabular}

AU, Assiut University; KU, Kyushu University; NWTS, National Wilm's Tumour Study; SIOP, International Society of Paediatric Oncology; SECI, South Egypt Cancer Institute.

hospital and received preoperative chemotherapy according to the SIOP-based protocol. There were 40 males and 39 females. The male to female ratio was $1: 1$.

Their ages ranged from 7 months to 5 years, mean age 24 months. Forty seven $(59.5 \%)$ of the patients were below 2 years at the time of diagnosis. The modes of presentation of patients with Wilm's tumour in this group are shown in Table 1, 85\% of the patients presented with an abdominal mass and/or distention. In thirty (38\%) patients, the Wilm's tumour was located in the right kidney, in $43(54.4 \%)$ patients, the Wilm's tumour was located on the left side and in six (7.6\%) patients, synchronous Wilm's Tumours were reported.

The distribution of the clinical stage (before and after chemotherapy) was as follows: $\mathrm{I}=25.3 \% \rightarrow 50 \%$, II $=$ $19 \% \rightarrow 20 \%, \quad$ III $=35.4 \rightarrow 15 \%, \quad$ IV $=12.6 \% \rightarrow 5 \% \quad$ and $\mathrm{V}=7.6 \% \rightarrow 7.6 \%$ (Fig. 1); there was an increase in the number of patients with stage I disease after chemotherapy. The pathology is shown in Table 1 .

Incomplete resection was encountered in three patients (4\%) and relapse occurred in four $(5 \%)$. With a median follow-up of 70 months, the 5-year overall survival was $84 \%$. When all patients are considered (without exclusion of patients lost follow up) and when abandonment included the overall survival was $80 \%$.

The 5-year stage-related survival was as follows: stage I (92\%), stage II $(80 \%)$, stage III $(50 \%)$, stage IV $(31 \%)$ and stage $\mathrm{V}(30 \%)$.
Fig. 1

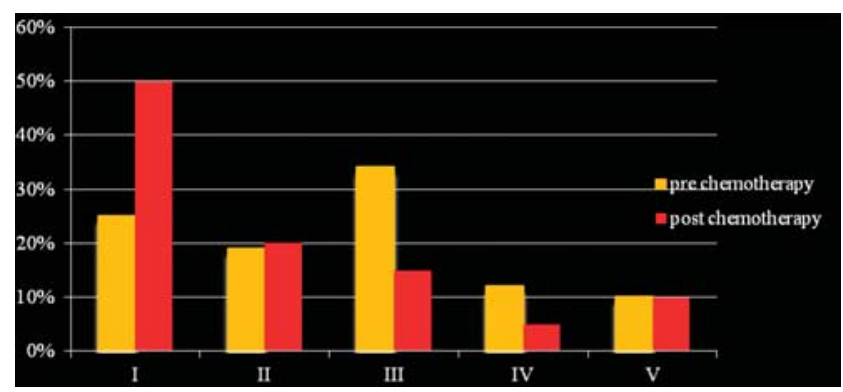

Prechemotherapy and postchemotherapy staging of South Egypt Cancer Institute group.

\section{Assiut University group data}

During the period from January 2000 to January 2009, 33 patients were treated at the Pediatric Surgery Department of Assiut University Hospital according to the NWTS protocol. The age of the patients ranged from 1-9 years; $17(51.5 \%)$ patients were below 2 years at the time of diagnosis. The mean age at diagnosis of patients was 2.5 years; the male to female ratio was $1: 1.2$. The tumour was localized in the left kidney in 13 (39.4\%) patients, the right kidney in $17(51.5 \%)$ patients and a synchronous bilateral Wilm's tumour was present in three (9\%) patients. The most common presenting symptom at diagnosis was an abdominal mass and/or abdominal distension, $82 \%$. The distribution of the clinical stage was as follows: stage I (33.3\%), stage II (21.2\%), stage III $(27.3 \%)$, stage IV (12\%) and stage V (6\%).

Intraoperative tumour rupture was encountered in seven (21\%) patients; three of these developed recurrence. Incomplete resection was reported in $10(30 \%)$ patients; four of these developed recurrence.

The pathological diagnosis is shown in Table 2. Postoperative recurrence was reported in seven (21.2\%) patients. The 5 -year overall survival was $77 \%$. The 5 -year stage-related survival as follows: $\mathrm{I}=85 \%, \mathrm{II}=75 \%$, III $=$ $52 \%, \mathrm{IV}=33 \%$ and $\mathrm{V}=33 \%$.

\section{Discussion}

Wilm's tumour is the most common embryonic malignancy of renal origin [6]. The survival rate of children with Wilm's tumours has improved considerably since the prospective randomized trials conducted by the NW'TSG, SIOP and the UKCCSG. Although the overall management of children with Wilm's tumours is one of the great success stories in cancer therapy and currently more than $90 \%$ of children survive 4 years after their diagnosis, there is still room for improvement [7].

The median age in our series was 3, 2.5 and 2 years for the $\mathrm{KU}$ group, the AU group and the SECI group, respectively, which is almost the same as that reported in other series [8-15]. More than 59.5\% of SECI patients, $47.5 \%$ of KU patients and $51.5 \%$ of AU patients are below 2 years at presentation, whereas $95,72.5$ and $84.8 \%$ of SECI, KU and AU groups, respectively, are below 4 years at 
Table 2 Preoperative chemotherapy regimen for the South Egypt Cancer Institute group

\begin{tabular}{llllllll}
\hline & \multicolumn{7}{c}{ Week } \\
\cline { 2 - 7 } & 1 & 2 & 3 & 4 & 5 & 6 \\
\hline Clinical and radiological stages I and II: two drugs for & 4 weeks \\
Vincristine $\left(1.4 \mathrm{mg} / \mathrm{m}^{2}\right.$, intravenously) & V & V & V & V & - & - \\
Dactinomycin $(45 \mu \mathrm{g} / \mathrm{kg}$, intravenously) & A & - & - & A & - & - \\
Clinical and radiological stages III, IV, V: for & 6 & weeks & & & & \\
Vincristine $\left(1.4 \mathrm{mg} / \mathrm{m}^{2}\right.$, intravenously) & V & V & V & V & V & V \\
Dactinomycin $\left(45 \mu \mathrm{g} / \mathrm{kg}_{\text {, intravenously) }}\right.$ & - & A & - & - & A & - \\
Doxoroubocin $\left(20 \mathrm{mg} / \mathrm{m}^{2}\right.$, intravenously) & - & D & - & - & D & -
\end{tabular}

Babies $<12$ months of age received one-half the recommended dose of all drugs. D, Dactinomycin; V, Vincristine.

presentation; these are in agreement with the results of Weirch et al. [16]. Although Grabois and Mendonca [17] and Carli et al. [18] reported age below 2 and 4 years as follow $22.7 \%, 55.3 \%$ and $11 \%, 74.2 \%$, respectively [16,17]. The age of Egyptian patients is younger than Japanese patients; this may be because of racial and genetic causes, but in both groups, the age of most patients is below 4 years, which is similar to that reported in previous studies.

The sex ratio in the current study was the same for both Japanese and Egyptian patients, which is similar to that reported in most other series [8,11,19-21], although the sex ratio reported from some developing countries shows a slight male predominance $[9,10,13,14]$.

The main presenting symptom of our study was an abdominal mass and/or distension, found in $72 \%$ of $\mathrm{KU}$ patients, which is similar to that reported in the first Wilm's tumour study of UKCCSG and a study from a South African Center; these rates were 74 and $77 \%$, respectively [22,23], however, in SECI patients and AU patients the abdominal mass as presenting symptoms are $85 \%$ and $82 \%$ respectively, these rates were similar to rate reported by other studies $[9,15]$. However, Yildiz et al. [8] (Turkey) and Madani et al. [10] (Morocco) reported a 95\% and $97 \%$ rate of presence of an abdominal mass as presenting symptoms, respectively. There is a higher incidence of an abdominal mass and/or distension in SECI and AU patients; this may be because of a delayed diagnosis.

The stage I percentage in our study was $50 \%$ for the SECI group (postchemotherapy), $40 \%$ for the $\mathrm{KU}$ group and $33.3 \%$ for the $\mathrm{AU}$ group; this difference was because of the preoperative chemotherapy treatment that was provided for SECI patients. The percentage of stage I for SECI patients is almost the same as that reported in Morocco by Madani et al. (50\%) [8] and slightly lower than the rate reported in some SIOP series, which is greater than $60 \%[19,24]$, but greater than other SIOP series, which is less than $40 \%[17,25]$. Sidhom et al. and Zaghloul et al. (National cancer Institute, Cairo University) reported a $21.4 \%$ and a $22.3 \%$ percentage of stage I in Egyptian patients, respectively, treated according to NWTS [26,27]. However, the percentages of stage I in $\mathrm{KU}$ and $\mathrm{AU}$ patients are similar to those in other studies reported by Pianezza et al. (Ontario, Canada) [15] and higher than those reported by Davidson et al. (South Africa) and Grabois et al. (Brazil): $30.3 \%$ and 19.7\%, respectively, on the basis of NWTS $[12,16]$. From the above result, we can conclude the importance of preoperative chemotherapy in downstaging of Wilm's tumours (downstage of higher stages to stage I so increase the incidence of stage I disease) subsequently easier surgery and can achieve complete surgical resection.

In our study, tumours were localized in the left kidney in $55 \%$ of patients and in 51.5 and $54.4 \%$ of Japanese and Egyptian patients, respectively; there was no statistically significant difference and this was similar to that in previous studies that reported that the left kidney was involved more frequently than the right kidney [10,28-32].

Three KU patients (7.5\%) and two AU patients (6\%) had a bilateral renal tumour. However, six SECI patients (7.6\%) had a bilateral renal tumour; previous studies have reported the incidence of bilateral Wilm's tumours to range from 4 to $10 \%[10,14,15,17,27,33-35]$.

Histopathologically, $17.5 \%$ of KU, $8.8 \%$ of SECI and $9 \%$ of AU patients had an unfavourable histology. In NWTS3, the rate of an unfavourable histology was $11.2 \%$ [36]. In contrast, in the SIOP-9/GPOH study, the rate of the presence of an anaplastic Wilm's tumour was 7.5\% [18]. There is a higher incidence of an unfavourable histology among Japanese patients; the exact cause for this is unknown, but a study carried out at Shanghai Children's Medical Center, China, reported that the incidence of Wilm's tumours with an unfavourable histology (including anaplasia, clear cell sarcoma and rhabdoid tumours) was $30 \%$.

Slightly more than $1 \%$ (one patient $1.3 \%$ ) of the patients in the SECI group received the wrong preoperative chemotherapy for benign disease (xanthogranulomatous pyelonephritis); SIOP studies have reported that, if a tumour has typical imaging and clinical features of a Wilm's tumour, chemotherapy should be started without histological confirmation. Thus, $1 \%$ of children with a noncancerous renal lesion receive chemotherapy [37]. The UKW3 trial reported that $12 \%$ of renal tumours with typical features of Wilm's tumours on imaging studies were found to be non-Wilm's tumours on a prechemotherapy biopsy [38]. On SIOP 93-01, approximately 5\% of lesions in patients treated with chemotherapy were ultimately found not to be Wilm's tumours and $1.8 \%$ of lesions were found to be benign [39]. Xanthogranulomatous pyelonephritis is rare in children and is difficult to diagnose preoperatively, especially when the signs of sepsis are not prominent [40]; also, preoperative chemotherapy may induce pathological changes similar to the pathology picture of xanthogranulomatous pyelonephritis. However, all patients in the KU and AU groups were accurately diagnosed histopathologically and a proper postoperative management was initiated.

In the SECI group, in patients treated by preoperative chemotherapy on the basis of the SIOP protocol, the incidence of intraoperative tumour rupture was $0 \%$, whereas in Japanese patients treated according to the NWTS protocol, the incidence was 15 and $21 \%$ in AU. The UKW3 reported that there were no tumour ruptures among the 102 patients in the preoperative chemotherapy group compared with 15 (14.7\%) among the 103 patients randomized to immediate nephrectomy [41]. This result shows the importance of preoperative chemotherapy in minimizing intraoperative tumour spillage. 
In SECI patients, the incidence of incomplete resection was $10 \%$ and the rate of recurrence was $5 \%$. In the KU group, incomplete resection was encountered in nine $(22.5 \%)$ of 40 patients; three $(33.3 \%)$ of these patients died and two of them developed postoperative recurrence. Among 40 patients, seven $(17.5 \%)$ died during the course of follow-up and four (57\%) had an unfavourable histopathology (three cases of a rhabdoid tumour of the kidney and one case clear cell sarcoma of the kidney). Six of 40 patients (15\%) developed postoperative recurrence; five of these $(83 \%)$ patients died. This result is in agreement with the reports from early studies that reported that the long-term survival rate after recurrence of a Wilm's tumour hardly reached 30\% [42].

The intra operative tumor rupture increase incidence of local recurrence with its gloom outcome.

The 5-year overall survival rate for KU was 82.5 and $77 \%$ for AU patients. When all patients are considered (without exclusion of patients lost follow up) and when abandonment included the overall survival was $80 \%$. There was no statistically significant difference between the two groups.

The major advantages of preoperative chemotherapy in the treatment of Wilm's tumours are as follows: (i) a reduction in the dose of adjuvant chemotherapy as a result of preoperative downstaging of the tumour; (ii) a decrease in the incidence of intraoperative tumour ruptures; and (iii) a reduced need for postoperative radiotherapy in cases of metastatic disease. Besides its acute toxicity, the major disadvantage of preoperative chemotherapy is the lack of a valid histopathological diagnosis before beginning the treatment, with a consequent risk of treating benign lesions resistant to chemotherapy. However, this is estimated to be $1.5-5 \%$ and, therefore, is acceptable when balanced against the advantages of the strategy.

Although North America and Europe have different philosophies on preoperative chemotherapy, the overriding message is that most patients with Wilm's tumours survive in the long term, irrespective of the sequence of therapeutic interventions.

\section{Conclusion}

Egyptian patients with Wilm's tumours presented at a younger age and had a lower incidence of an unfavourable histology compared with Japanese patients. Preoperative chemotherapy increased the rate of stage I disease and decreased the incidence of intraoperative tumour rupture and relapse. However, there is a possibility that chemotherapy may have been administered in patients with benign disease.

\section{Acknowledgements Conflicts of interest}

There are no conflicts of interest.

\section{References}

1 Kumar S, Carr T, Marsden HB, Calabuig-Crespo MC. Study of childhood renal tumours using antisera to fibronectin, laminin, and epithelial membrane antigen. J Clin Pathol 1986; 39:51-57.

2 Stiller CA, Parkin DM. International variations in the incidence of childhood renal tumours. Br J Cancer 1990; 62:1026-1030.
3 Coppes MJ, De Kraker J, Van Dijken PJ, Perry HJM, Delemarre JFM, Tournade MF, et al. Bilateral Wilm's tumor: long-term survival and some epidemiological features. J Clin Oncol 1989; 7:310-315.

4 Nawaz A, Matta H, Jacobsz A, Shawis R, Mpofu C, Al-Salem A. Wilm's Tumor: the Tawam hospital experience. Ann Saudi Med 1999; 19:257-260.

5 D'Angio GJ, Breslow N, Beckwith JB, Evans A, Baum E, DeLorimier A, et al. Treatment of Wilm's tumor. Results of the third national Wilm's tumor study. Cancer 1989; 64:349-360.

6 Tournade MF, Com-Nougue C, Voute PA, Lemerle J, De Kraker J, Delemarre JFM, et al. Results of the sixth international society of pediatric oncology Wilm's tumor trial and study: a risk-adapted therapeutic approach in Wilm's tumor. J Clin Oncol 1993; 11:1014-1023.

7 Grosfeld JL. Risk-based management: current concepts of treating malignant solid tumors of childhood. J Am Coll Surg 1999; 189:407-425.

8 Madani A, Zafad S, Harif M, Yaakoubi M, Zamiati S, Sahraoui S, et al. Treatment of Wilms tumor according to SIOP 9 protocol in Casablanca, Morocco. Pediatr Blood Cancer 2006; 46:472-475.

9 Baez F, Bellani FF, Ocampo E, Conter V, Flores A, Gutierrez T, et al. Treatment of childhood Wilm's tumor without radiotherapy in Nicaragua. Ann Oncol 2002; 13:944-948.

10 Yildiz I, Yüksel L, Özkan A, Apak H, Celkan T, Danismend N, et al. Multidisciplinary approach to Wilm's tumor: 18 years of experience. Jpn J Clin Oncol 2000; 30:17-20.

11 Green DM, D'Angio GJ, Beckwith JB, Breslow NE, Grundy PE, Ritchey ML, Thomas PRM. Wilms tumor. CA Cancer J Clin 1996; 46:46-63.

12 Davidson A, Hartley P, Desai F, Daubenton J, Rode H, Millar A. Wilms tumour experience in a South African centre. Pediatr Blood Cancer 2006; 46: 465-471.

13 Mahmood A, Ghafoor T, Badsha S. Wilm's tumour: presentation and treatment. J Coll Physicians Surg Pak 2004; 14:142-145.

14 Hung I-J, Yang C-P, Jiang T-H. Clinical features of Wilm's tumor and treatment results. J Formos Med Assoc 1993; 92:816-822.

15 Pianezza ML, Rubin S, Bass J, Chou S, Pike JG, Leonard MP. Wilm's tumor at the Children's Hospital of Eastern Ontario: 1990-2001. Can J Urol 2004; 11:2151-2156.

16 Weirich A, Ludwig R, Graf N, Abel U, Leuschner I, Vujanic GM, et al. Survival in nephroblastoma treated according to the trial and study SIOP-9/GPOH with respect to relapse and morbidity. Ann Oncol 2004; 15:808-820.

17 Grabois MF, Mendonca GA. Prognosis for patients with unilateral Wilms' tumor in Rio de Janeiro, Brazil. 1990-2000. Rev Saude Publica 2005; 39:731-737.

18 Carli MFE, Frascella E, Tournade MF. Second malignant neoplasms in patients treated on SIOP Wilms tumour studies and trials 1, 2, 5, and 6 . Med Pediatr Oncol 1997; 29:239-244.

19 Reinhard H, Bode B, Burger D. Results of the SIOP 93-01/GPOH trial and study for the treatment of patients with unilateral nonmetastatic Wilms Tumor. Klin Padiatr 2004; 216:132-140.

20 Koesters RNF, Von Knebel Doeberitz M. Nuclear accumulation of beta-catenin protein in Wilms' tumours. J Pathol 2003; 199:68-76.

21 McNally RJ, Alston RD, Cairns DP. Geographical and ecological analyses of childhood Wilms' tumours and soft-tissue sarcomas in North West England. Eur J Cancer 2003; 39:1586-1593.

22 Pritchard J, Imeson J, Barnes J. Results of the United Kingdom Children's Cancer Study Group first Wilms' Tumor Study. J Clin Oncol 1995; 13:124-133.

23 Tournade MF, Com-Nougué C, De Kraker J. Optimal duration of preoperative therapy in unilateral and nonmetastatic Wilms' tumor in children older than 6 months: results of the Ninth International Society of Pediatric Oncology Wilms' Tumor Trial and study. J Clin Oncol 2001; 19:488-500.

24 Seseke FZG, Zöller G, Ringert RH. Wilms' tumor-single-center experience with renal surgery. Scand J Urol Nephrol 2004; 38:373-377.

25 Sidhom HH, Kotb G, Anwer G. National Cancer Institute, Cairo University, Egypt; Aboul Reish Hospital, Cairo University, Egypt, Multidisciplinary Approach to Wilms' Tumor: 10 years Experience of $\mathrm{NCl}$, . Egypt. J Clin Oncol, Meeting Proceedings (Post-meeting Edition) 2004. 22 (No 14S (July 15 Supplement)) abstract No: 8544.

26 Zaghloul MS, Hussein MH, El Koutbey M. Wilms' tumor: long-term results from a single institution. J Surg Oncol 1994; 56:25-31.

27 Ehrlich PF, Ritchey ML, Hamilton TE. Quality assessment for Wilms' tumor: a report from the National Wilms' Tumor Study-5. J Pediatr Surg 2005; 40:208-12, discussion 212-213.

28 De Kraker J, Graf N, van Tinteren H. Reduction of postoperative chemotherapy in children with stage I intermediate-risk and anaplastic Wilms' tumour (SIOP 93-01 trial): a randomised controlled trial. Lancet 2004; 364:1229-1235

29 Agaoglu L, Unuvar A, Anak S. A twenty-one year experience with 120 Wilms' tumor cass. Med Pediatr Oncol 1997; 29:449.

30 Lemerle J, Tournade F, Marchant RG. Wilms' tumor: natural history and prognostic factors: a retrospective study of 248 cases treated at the Institut Gustave-Roussy 1952-1967. Cancer 1976; 37:2557-2566.

31 Jenkner A, Df Boldrini R. 111 Renal Neoplasms of Childhood: a Clinicopathologic Study. J Pediatr Surg 2001; 36:1522-1527. 
32 Gommersall LM, Arya M, Mushtaq I. Current challenges in Wilms' tumor management. Nat Clin Pract Oncol 2005; 2:298-304, quiz 1 p following 324.

33 Millar AJ, Davidson A, Rode H. Bilateral Wilms' tumors: a single-center experience with 19 cases. J Pediatr Surg 2005; 40:1289-1294.

34 Montgomery BT, Kelalis PP, Blute ML. Extended followup of bilateral Wilms tumor: results of the National Wilms Tumor Study. J Urol 1991; 146 (2) (Pt 2):514-518.

35 Pritchard-Jones K. Controversies and advances in the management of Wilms' tumour. Arch Dis Child 2002; 87:241-244.

36 Vujanic GM, Kelsey A, Mitchell C. The role of biopsy in the diagnosis of renal tumors of childhood: results of the UKCCSG Wilms tumor study 3. Med Pediatr Oncol 2003; 40:18-22.

37 Samuel M, Duffy P, Capps S. Xanthogranulomatous pyelonephritis in childhood. J Pediatr Surg 2001; 36:598-601.
38 Pritchard-Jones K. Controversies and advances in the management of Wilms' tumour. Arch Dis Child 2002; 87:241-244.

39 Groot-Loonen JJ, Pinkerton CR, Morris-Jones PH. How curable is relapsed Wilms' tumour? The United Kingdom Children's Cancer Study Group. Arch Dis Child 1990; 65:968-970.

40 Grundy P, Breslow N, Green DM. Prognostic factors for children with recurrent Wilms' tumor: results from the Second and Third National Wilms' Tumor Study. J Clin Oncol 1989; 7:638-647.

41 Pinkerton CR, Groot-Loonen JJ, Morris-Jones PH. Response rates in relapsed Wilms' tumor. a need for new effective agents. Cancer 1991; 67:567-571.

42 Wilimas JA, Champion J, Douglass EC. Relapsed Wilms' tumor. Factors affecting survival and cure. Am J Clin Oncol 1985; 8:324-328. 